\title{
Magnetic Dioxygen Clathrate Hydrates: A Type of Promising Building Blocks for Icy Crystalline Materials
}

Hongshu Zhang, Haibei Huang, Liang Gao, Qiang Fu, Shibo Cheng, Yuxiang Bu* School of Chemistry and Chemical Engineering, Shandong University, Jinan 250100, People's Republic of China

\section{Supporting Information}
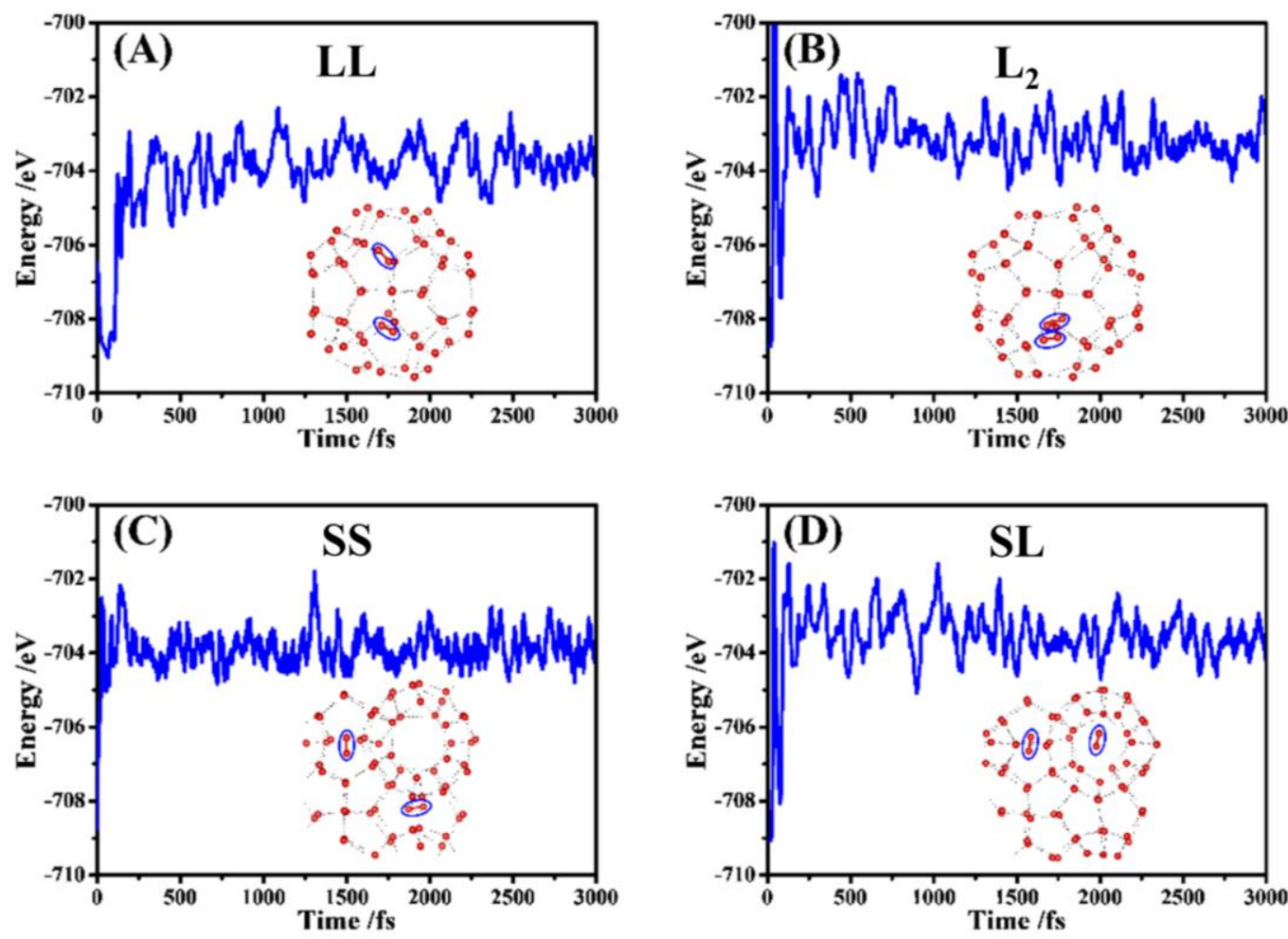

Figure S1. Time evolution of total energies with respect to the evolution time for $\operatorname{LL}(A), L_{2}(B)$, SS (C), and SL (D).

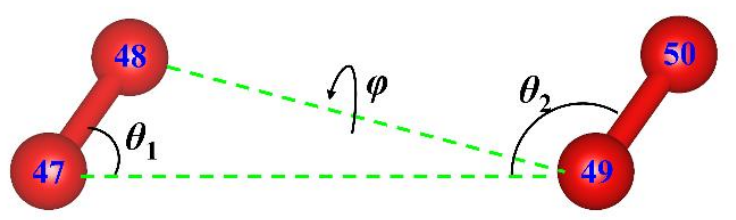

Figure S2. Relative orientations of two guest $\mathrm{O}_{2}$ molecules with geometrical parameters $(\mathrm{H}$ orientation: $\theta_{1}=\theta_{2}=90^{\circ}, \varphi=0^{\circ}$; T orientation: $\theta_{1}=85^{\circ}, \theta_{2}=175^{\circ}, \varphi=0^{\circ} ;$ X orientation: $\theta_{1}=\theta_{2}$ $\left.=85^{\circ}, \varphi=90^{\circ}\right)$ 

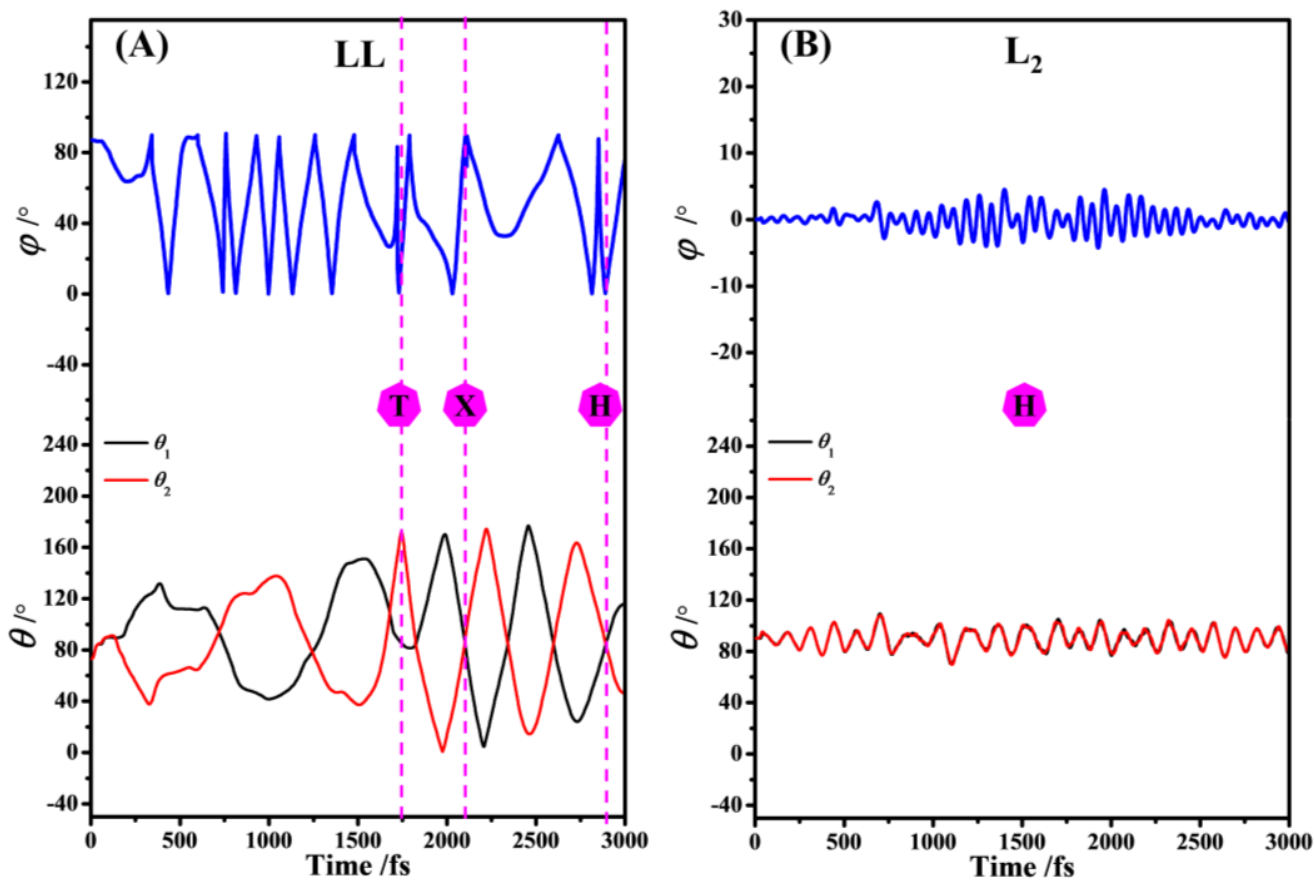

Figure S3. The fluctuations of dihedral angle $\varphi$ and angle $\theta$ as a function of the evolution time in ab initio molecular dynamic simulations for two $\mathrm{O}_{2}$ molecules in LL (A) and $\mathrm{L}_{2}(\mathrm{~B})$.
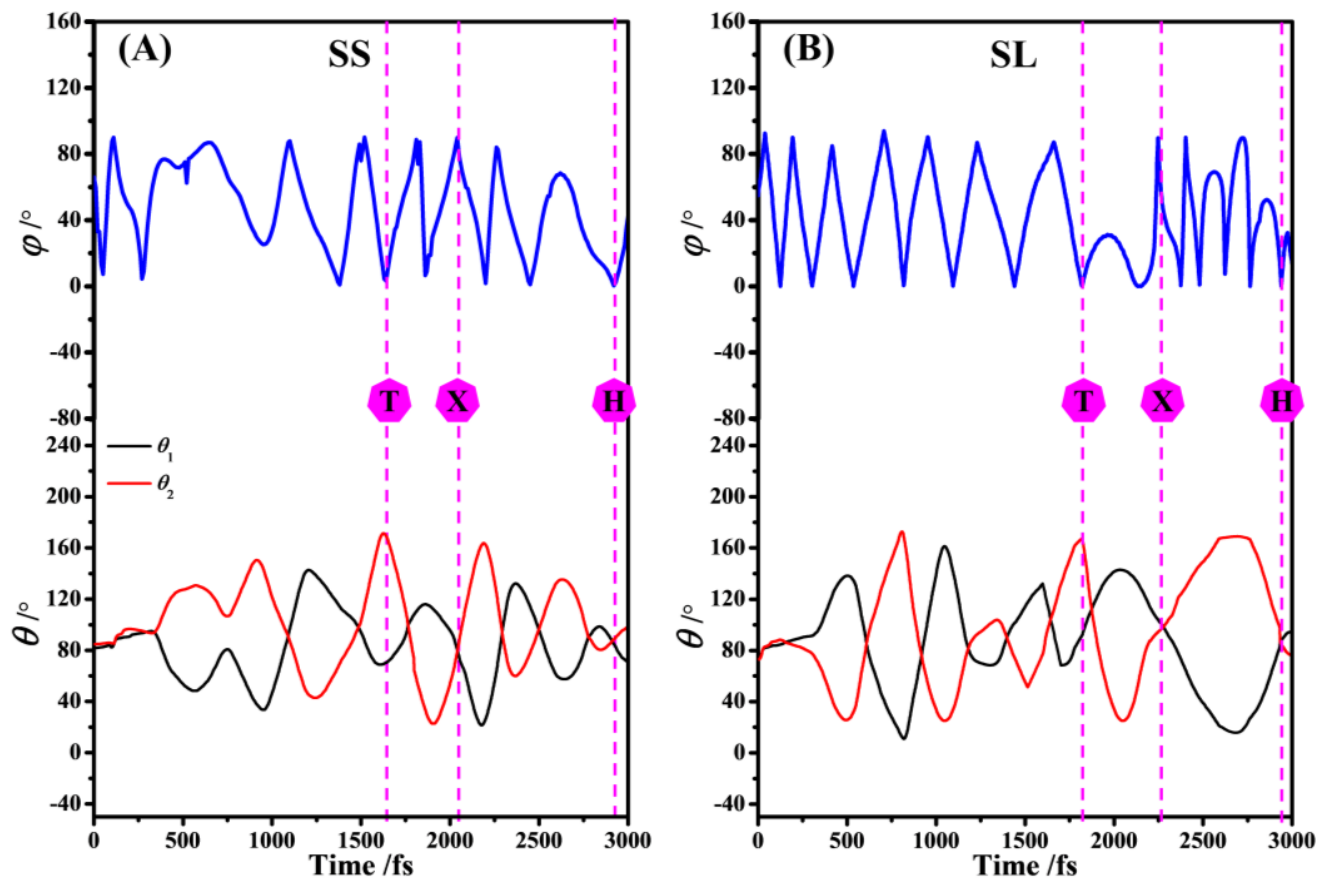

Figure S4. The fluctuations of dihedral angle $\varphi$ and angle $\theta$ as a function of the evolution time in ab initio molecular dynamic simulations for two $\mathrm{O}_{2}$ molecules in SS (A) and SL (B). 


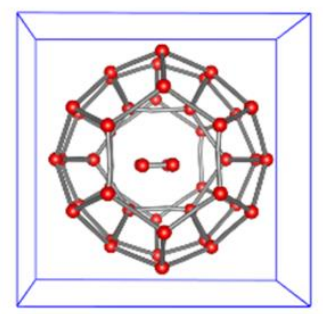

${ }^{5} \mathbf{L}^{6} \mathbf{L} / \mathbf{H}$

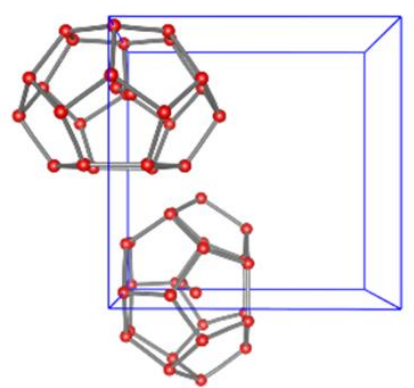

${ }^{1} \mathbf{L}^{3} \mathbf{L} / \mathbf{H}$

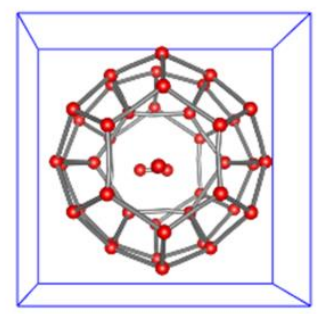

${ }^{5} \mathbf{L}^{6} \mathbf{L} / \mathbf{T}$

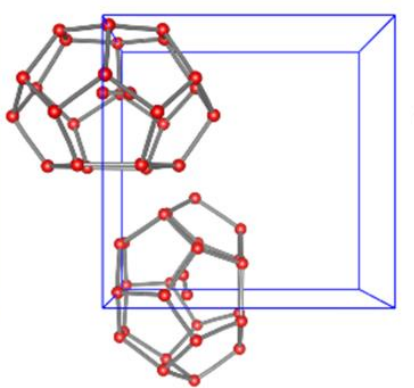

${ }^{1} \mathbf{L}^{3} \mathbf{L} / \mathbf{T}$

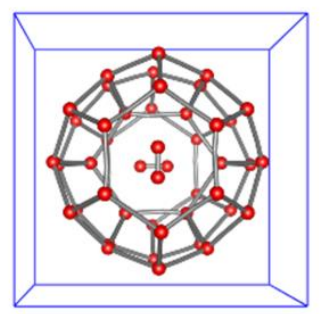

${ }^{5} \mathbf{L}^{6} \mathbf{L} / \mathbf{X}$

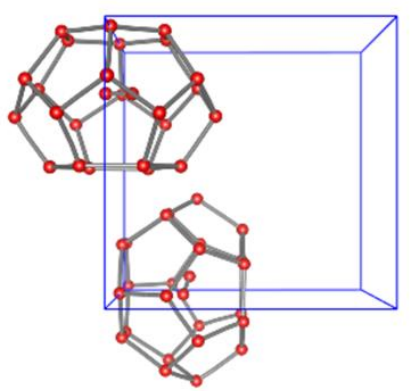

${ }^{1} \mathbf{L}^{3} \mathbf{L} / \mathbf{X}$

Figure S5. Three optimized orientations (parallel/H, vertical/T, and crossed/X) of two guest $\mathrm{O}_{2}$ molecules in the ${ }^{5} \mathrm{~L}^{6} \mathrm{~L}$ or ${ }^{1} \mathrm{~L}^{3} \mathrm{~L}$ structures. Only the $\mathrm{H}_{2} \mathrm{O} \mathrm{O}$ atoms are displayed in the cage framework and all $\mathrm{H}$ atoms are omitted. Similarly, only the cages which include $\mathrm{O}_{2}$ guest molecules are displayed in the crystal cell, and the other cages are omitted.

Table S1. The Calculated NM, FM, and AFM Magnetic State Energies ( $E_{\mathrm{NM}}, \mathrm{E}_{\mathrm{FM}}, \mathrm{E}_{\mathrm{AFM}}$ ), and Distances between Two Guest $\mathrm{O}_{2}$ Molecules $\left(d_{\mathrm{O} 2-\mathrm{O} 2}\right)$ for the ${ }^{5} \mathrm{~L}{ }^{6} \mathrm{~L}(\mathrm{H}, \mathrm{T}$, and $\mathrm{X})$ and ${ }^{1} \mathrm{~L}{ }^{3} \mathrm{~L}(\mathrm{H}, \mathrm{T}$, and $\mathrm{X}$ ) structures

\begin{tabular}{cccccc}
\hline Structures & Orientations & $\mathbf{E}_{\mathbf{N M}} / \mathbf{e V}$ & $\mathbf{E F M}_{\mathbf{F M}} / \mathbf{e}$ & $\mathbf{E}_{\text {AFM/eV }}$ & $\boldsymbol{d o 2}_{\mathbf{O}-\mathbf{O} 2} / \mathbf{A}$ \\
\hline \multirow{3}{*}{${ }^{\mathbf{6}} \mathbf{6} \mathbf{L}$} & $\mathrm{H}$ & -707.100 & -08.678 & -708.684 & 5.675 \\
& $\mathrm{~T}$ & -707.093 & -708.669 & -708.669 & 5.695 \\
& $\mathrm{X}$ & -707.098 & -708.650 & -708.650 & 5.688 \\
${ }^{1} \mathbf{L}^{3} \mathbf{L}$ & $\mathrm{H}$ & -707.096 & -708.669 & -708.669 & 10.830 \\
& $\mathrm{~T}$ & -707.091 & -708.672 & -708.672 & 11.456 \\
& $\mathrm{X}$ & -707.096 & -708.678 & -708.678 & 11.131 \\
\hline
\end{tabular}




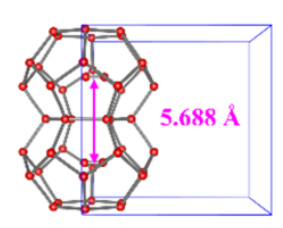

${ }^{1} \mathbf{L}^{2} \mathbf{L}$

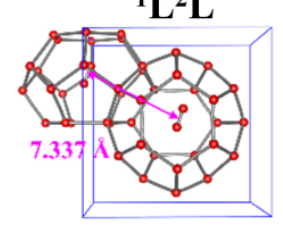

${ }^{1} \mathbf{L}^{5} \mathbf{L}$

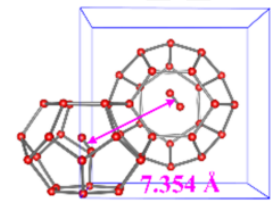

${ }^{2} \mathbf{L}^{6} \mathbf{L}$

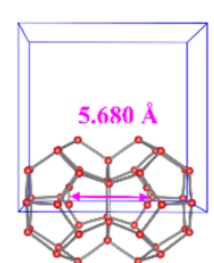

${ }^{3} \mathbf{L}^{4} \mathbf{L}$

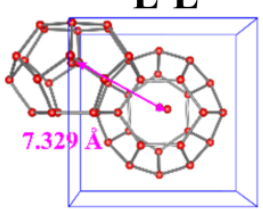

${ }^{1} \mathbf{L}^{6} \mathbf{L}$

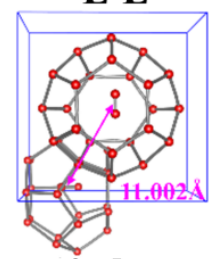

${ }^{3} \mathbf{L}^{5} \mathbf{L}$

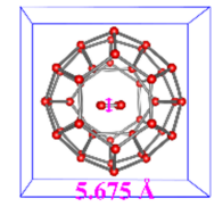

${ }^{5} \mathbf{L}^{6} \mathbf{L}$

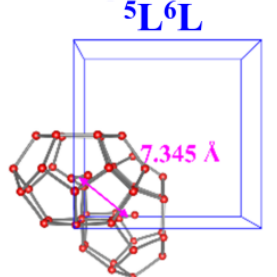

${ }^{2} \mathbf{L}^{3} \mathbf{L}$

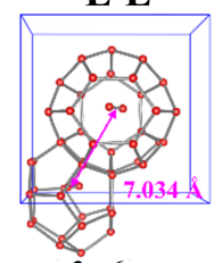

${ }^{3} \mathbf{L}^{6} \mathbf{L}$

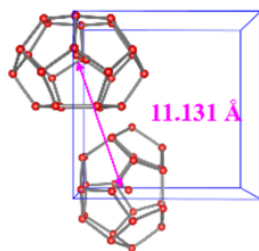

${ }^{1} \mathbf{L}^{3} \mathbf{L}$

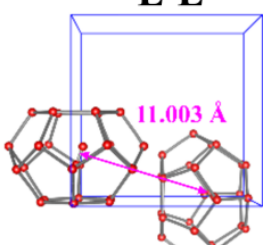

${ }^{2} \mathbf{L}^{4} \mathbf{L}$

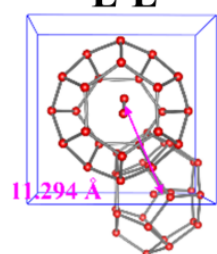

${ }^{4} \mathbf{L}^{5} \mathbf{L}$

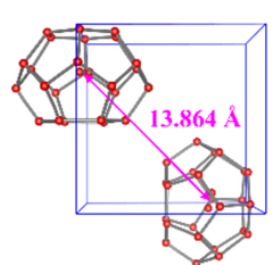

${ }^{1} \mathbf{L}^{4} \mathbf{L}$

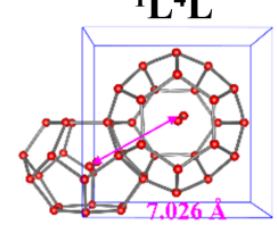

${ }^{2} L^{5} \mathbf{L}$

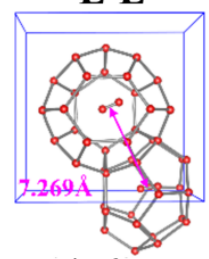

${ }^{4} \mathbf{L}^{6} \mathbf{L}$

Figure S6. The optimized geometries of two guest $\mathrm{O}_{2}$ molecules in two $\mathrm{L}$ cages. The most stable structure is ${ }^{5} \mathrm{~L}^{6} \mathrm{~L}$. The other cages and all hydrogen atoms in the cell are omitted.

Table S2. The Calculated $\mathrm{E}_{\mathrm{NM}}, \mathrm{E}_{\mathrm{FM}}$, and $\mathrm{E}_{\mathrm{AFM}}$ for $15 \mathrm{LL}$ structures with two $\mathrm{O}_{2}$ molecules encaged in different $\mathrm{L}$ cages

\begin{tabular}{ccccc}
\hline Structures & Orientations & $\mathbf{E N M}_{\mathbf{N M}} \mathbf{e V}$ & $\mathbf{E}_{\mathbf{F M}} / \mathbf{e V}$ & $\mathbf{E}_{\mathbf{A F M}} / \mathbf{e V}$ \\
\hline${ }^{1} \mathbf{L}^{2} \mathbf{L}$ & $\mathrm{H}$ & -706.667 & -708.678 & -708.683 \\
${ }^{3} \mathbf{L}^{4} \mathbf{L}$ & $\mathrm{H}$ & -706.663 & -708.677 & -708.682 \\
${ }^{5} \mathbf{L}^{6} \mathbf{L}$ & $\mathrm{H}$ & -706.668 & -708.678 & -708.684 \\
${ }^{1} \mathbf{L}^{3} \mathbf{L}$ & $\mathrm{X}$ & -706.663 & -708.678 & -708.678 \\
${ }^{2} \mathbf{L}^{3} \mathbf{L}$ & $\mathrm{H}$ & -706.641 & -708.668 & -708.668 \\
${ }^{2} \mathbf{L}^{4} \mathbf{L}$ & $\mathrm{X}$ & -706.636 & -708.646 & -708.646 \\
${ }^{1} \mathbf{L}^{4} \mathbf{L}$ & $\mathrm{X}$ & -706.654 & -708.675 & -08.675 \\
${ }^{1} \mathbf{L}^{5} \mathbf{L}$ & $\mathrm{H}$ & -706.648 & -708.668 & -708.668 \\
${ }^{2} \mathbf{L}^{5} \mathbf{L}$ & $\mathrm{H}$ & -706.645 & -708.667 & -708.667 \\
${ }^{3} \mathbf{L}^{6} \mathbf{L}$ & $\mathrm{H}$ & -706.641 & -708.661 & -708.661 \\
${ }^{4} \mathbf{L}^{6} \mathbf{L}$ & $\mathrm{H}$ & -706.640 & -708.646 & -708.646 \\
${ }^{1} \mathbf{L}^{6} \mathbf{L}$ & $\mathrm{H}$ & -706.636 & -708.650 & -708.650 \\
${ }^{2} \mathbf{L}^{6} \mathbf{L}$ & $\mathrm{H}$ & -706.647 & -708.668 & -708.668 \\
${ }^{3} \mathbf{L}^{5} \mathbf{L}$ & $\mathrm{X}$ & -706.640 & -708.665 & -708.665 \\
& & $\mathrm{~S} 4$ & &
\end{tabular}




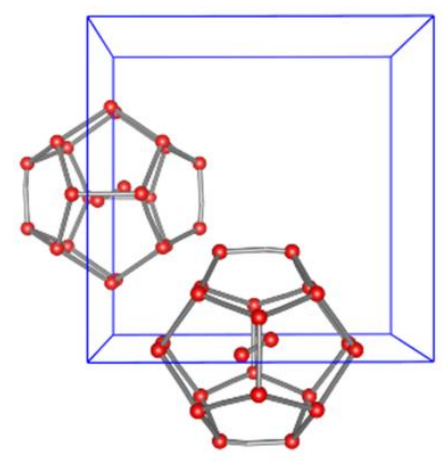

${ }^{a} \mathbf{S}^{b} \mathbf{S} / \mathbf{H}$

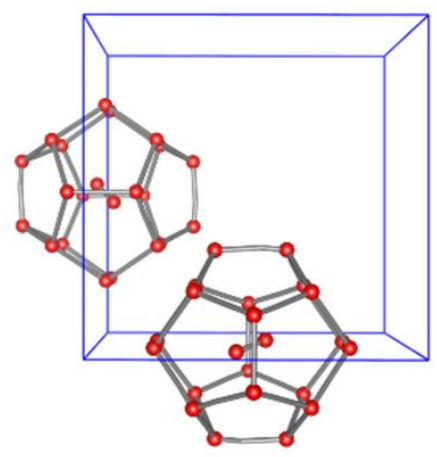

${ }^{\mathrm{a}} \mathbf{S}^{\mathrm{b}} \mathbf{S} / \mathbf{T}$

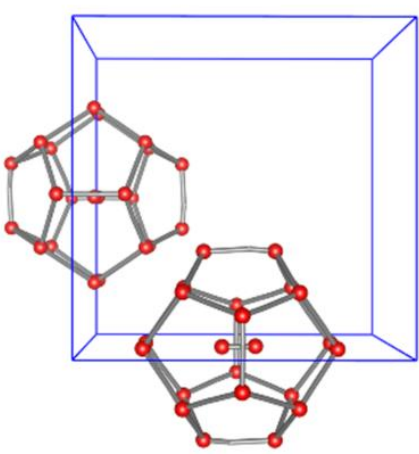

${ }^{\mathrm{a}} \mathbf{S}^{\mathrm{b}} \mathbf{S} / \mathbf{X}$

Figure S7. Three optimized orientations (H, T, and X) of two guest $\mathrm{O}_{2}$ molecules in two $\mathrm{S}$ cages. The $\mathrm{X}$-orientations is the most stable geometry configurations. The other cages and all hydrogen atoms in the cell are omitted.

Table S3. The Calculated $\mathrm{E}_{\mathrm{NM}}, \mathrm{E}_{\mathrm{FM}}$, and $\mathrm{E}_{\mathrm{AFM}}$ for the SS structures with different orientations of two $\mathrm{O}_{2}$ molecules in the $\mathrm{S}$ cages

\begin{tabular}{cccccc}
\hline Structures & Orientations & $\mathbf{E}_{\mathbf{N F}} / \mathbf{e V}$ & $\mathbf{E}_{\mathbf{F M}} / \mathbf{e V}$ & $\mathbf{E}_{\mathrm{AFM}} / \mathbf{e V}$ & $\boldsymbol{d}_{\mathbf{O} 2-\mathbf{0} 2 / \mathbf{A}}$ \\
\hline \multirow{3}{*}{${ }^{\mathbf{a}} \mathbf{S}^{\mathbf{b} S}$} & $\mathrm{H}$ & -706.721 & -708.672 & -708.672 & 10.523 \\
& $\mathrm{~T}$ & -706.661 & -708.673 & -708.671 & 10.511 \\
& $\mathrm{X}$ & -706.672 & -708.675 & -708.674 & 10.583 \\
\hline
\end{tabular}



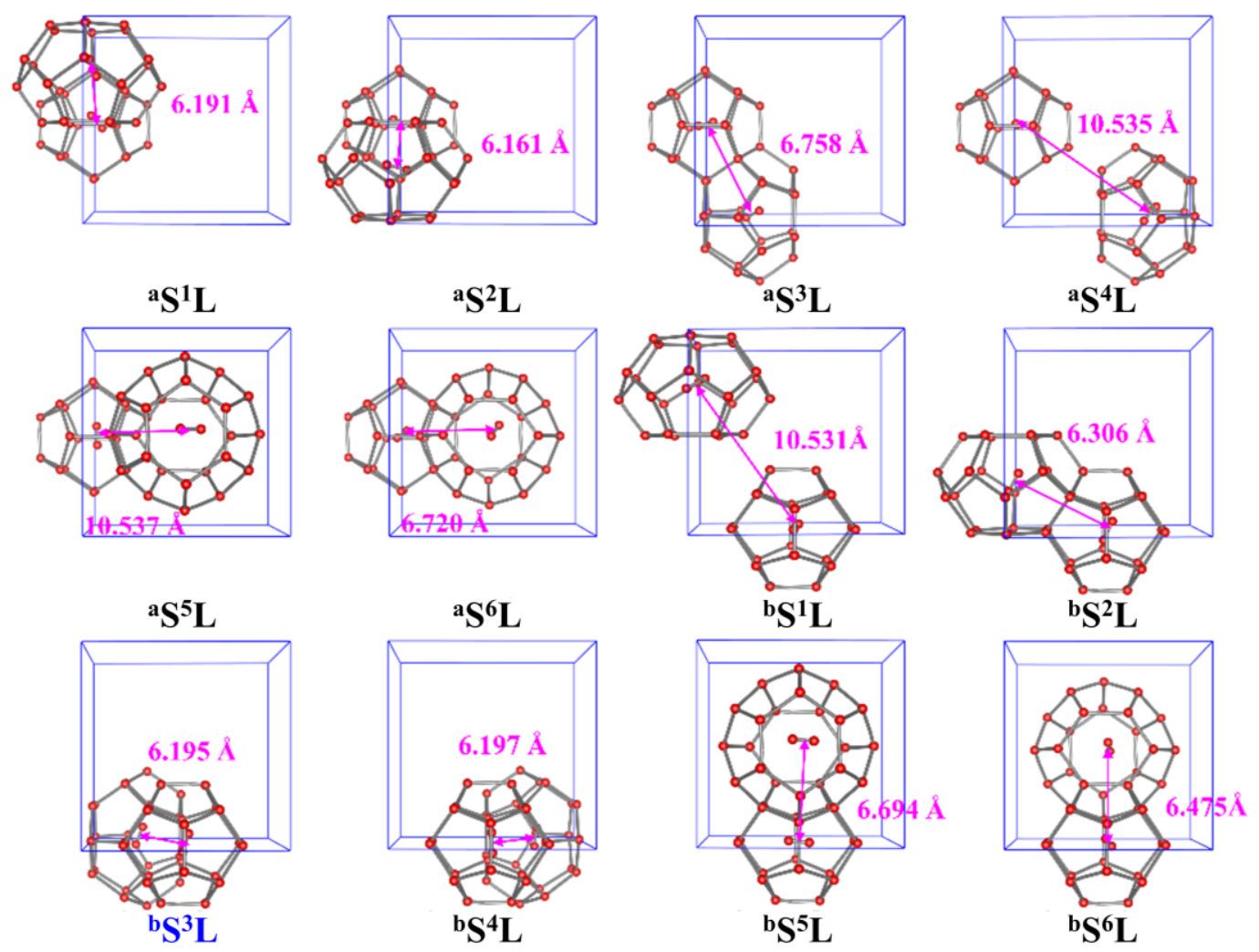

Figure S8. The optimized geometries of two guest $\mathrm{O}_{2}$ molecules in the $\mathrm{S}$ and L cages. The most stable structure is ${ }^{b} S^{3} \mathrm{~L}$. The other cages and all hydrogen atoms in the cell are omitted.

Table S4. The Calculated $E_{\mathrm{NM}}, \mathrm{E}_{\mathrm{FM}}$, and $\mathrm{E}_{\mathrm{AFM}}$ for the SL Structures with Two $\mathrm{O}_{2}$ Molecules Encaged in Different L and S Cages

\begin{tabular}{|c|c|c|c|c|}
\hline Structures & Orientations & $\mathrm{E}_{\mathrm{NM}} / \mathrm{eV}$ & $\mathrm{E}_{F M / e V}$ & $\mathrm{E}_{\mathrm{AFM}} / \mathrm{eV}$ \\
\hline${ }^{a} \mathbf{S}^{1} \mathbf{L}$ & $\mathrm{H}$ & -706.666 & -708.667 & -708.667 \\
\hline${ }^{\mathrm{a}} \mathbf{S}^{2} \mathbf{L}$ & $\mathrm{H}$ & -706.663 & -708.671 & -708.671 \\
\hline${ }^{a} \mathbf{S}^{3} \mathbf{L}$ & $\mathrm{H}$ & -706.659 & -708.665 & -708.665 \\
\hline${ }^{\mathrm{a}} \mathbf{S}^{4} \mathbf{L}$ & $X$ & -706.668 & -708.672 & -708.672 \\
\hline${ }^{a} S^{5} \mathbf{L}$ & $\mathrm{X}$ & -706.667 & -708.670 & -708.670 \\
\hline${ }^{\mathrm{a}} \mathbf{S}^{6} \mathbf{L}$ & $\mathrm{H}$ & -706.667 & -708.671 & -708.671 \\
\hline${ }^{b} \mathbf{S}^{1} \mathbf{L}$ & $\mathrm{X}$ & -706.668 & -708.670 & -708.670 \\
\hline${ }^{b} \mathbf{S}^{2} \mathbf{L}$ & $\mathrm{H}$ & -706.665 & -708.668 & -708.668 \\
\hline${ }^{b} \mathbf{S}^{3} \mathbf{L}$ & $\mathrm{H}$ & -706.670 & -708.673 & -708.673 \\
\hline${ }^{b} \mathbf{S}^{4} \mathbf{L}$ & $\mathrm{H}$ & -706.670 & -708.672 & -708.672 \\
\hline${ }^{b} \mathbf{S}^{5} \mathbf{L}$ & $\mathrm{H}$ & -706.664 & -708.666 & -708.666 \\
\hline${ }^{b} \mathbf{S}^{6} \mathbf{L}$ & $\mathrm{H}$ & -706.662 & -708.664 & -708.664 \\
\hline
\end{tabular}




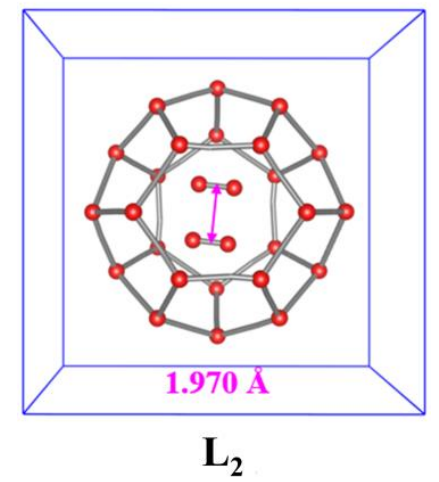

Figure S9. The optimized geometry configurations of two guest $\mathrm{O}_{2}$ molecules in one $\mathrm{L}$ cage. The other cages and all hydrogen atoms in the cell are omitted.

Table S5. The Calculated Lattice Constants $(a), \mathrm{O}_{2}$ Bond Lengths $\left(l_{\mathrm{O} 2}\right), \mathrm{E}_{\mathrm{NM}}, \mathrm{E}_{\mathrm{FM}}, \mathrm{E}_{\mathrm{AFM}}$, and Formation Energies ( $E_{\text {form}}$ ) for the ${ }^{5} \mathrm{~L}^{6} \mathrm{~L},{ }^{\mathrm{a}} \mathrm{S}{ }^{\mathrm{b}} \mathrm{S},{ }^{\mathrm{b}} \mathrm{S}{ }^{3} \mathrm{~L}$, and $\mathrm{L}_{2}$ Structures

\begin{tabular}{|c|c|c|c|c|c|c|}
\hline & $a / \AA$ & $l 02 / \AA ̊$ & $\mathrm{E}_{\mathrm{NF}} / \mathrm{eV}$ & EFM /eV & $\mathbf{E}_{\text {AFM }} / \mathrm{eV}$ & $E_{\text {form }} / \mathbf{e V}$ \\
\hline${ }^{5} \mathbf{L}^{6} \mathbf{L}$ & 11.396 & 1.233 & -707.100 & -708.678 & -708.684 & -0.236 \\
\hline${ }^{\mathrm{a}} \mathbf{S}^{\mathrm{b}} \mathbf{S}$ & 11.430 & 1.233 & -706.672 & -708.675 & -708.674 & -0.227 \\
\hline${ }^{b} \mathbf{S}^{3} \mathbf{L}$ & 11.413 & 1.236 & -706.670 & -708.673 & -708.673 & -0.225 \\
\hline $\mathbf{L}_{2}$ & 11.440 & 1.231 & -708.259 & -708.448 & -708.636 & -0.188 \\
\hline
\end{tabular}




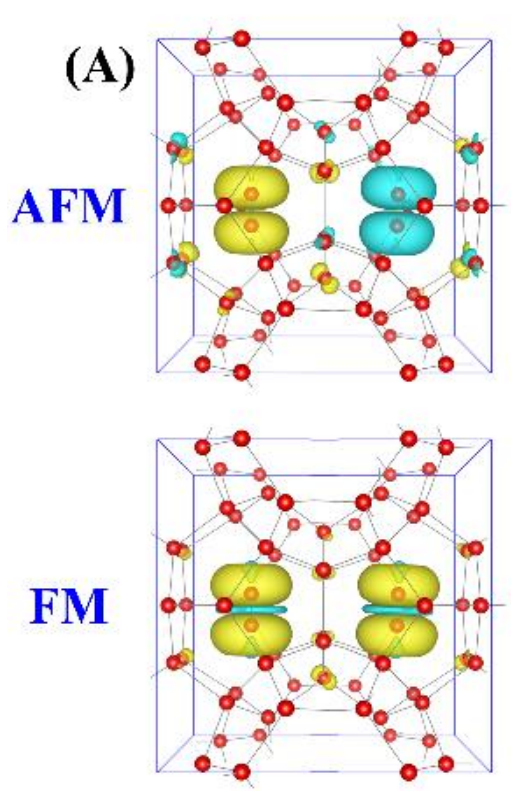

$\mathbf{H}$
(B)
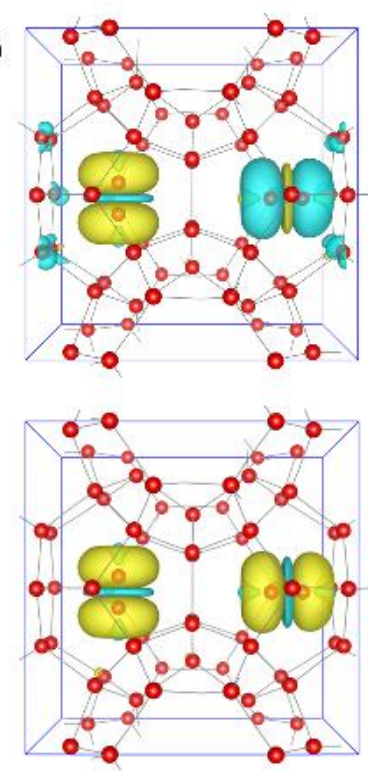

$\mathbf{T}$
(C)
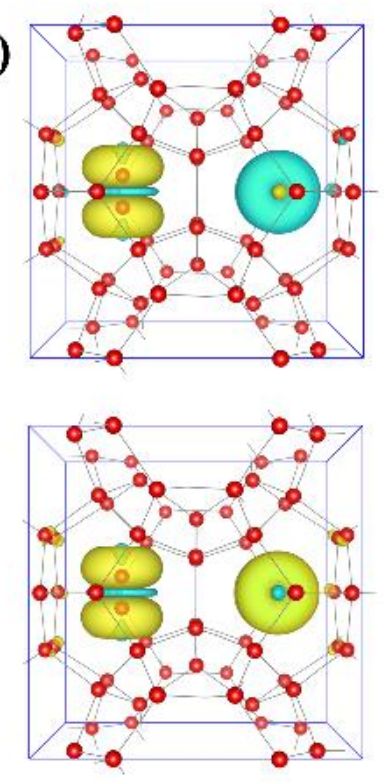

$\mathbf{X}$

Figure S10. Spin-polarized charge density distributions of the ${ }^{5} \mathrm{~L}^{6} \mathrm{~L}$ structures at different $d_{\mathrm{O} 2-\mathrm{O} 2}$ for three different geometry configurations (X, T, and $\mathrm{H})$.

Table S6. The Calculated Single Point Energies $\left(\mathrm{E}_{\mathrm{CS}} / \mathrm{E}_{\mathrm{BS}} / \mathrm{E}_{\mathrm{Q}}\right)$ Combined With the Average Spin Square Values $\left.\left(<\mathrm{S}^{2}\right\rangle\right)$ for the ${ }^{5} \mathrm{~L}^{6} \mathrm{~L}(\mathrm{H}, \mathrm{T}, \mathrm{X}), \mathrm{L}_{2} / \mathrm{H},{ }^{\mathrm{a}} \mathrm{S}^{\mathrm{b}} \mathrm{S} / \mathrm{X}$, and ${ }^{\mathrm{b}} \mathrm{S}^{3} \mathrm{~L} / \mathrm{H}$ Structures at the B3LYP/6-31+G(d) Level by using Ice as Medium (Dielectronic Constant 13.2) and the Polarizable Continuum Model (PCM). CS: Closed-Shell Singlet; BS: Broken-Symmetry OpenShell Singlet; Q: Quintuplet

\begin{tabular}{cccc}
\hline Structures & Ecs /a.u. & $\mathbf{E}_{\mathbf{B S}} / \mathbf{a . u} .\left\langle\mathbf{S}^{\mathbf{2}}\right\rangle$ & $\mathbf{E}_{\mathbf{Q}} / \mathbf{a . u} .\left\langle\mathbf{S}^{\mathbf{2}}\right\rangle$ \\
\hline${ }^{\mathbf{5}} \mathbf{L}^{\mathbf{6}} \mathbf{L} / \mathbf{H}$ & -9167.9203683 & -9168.0414298 & -9168.0412556 \\
${ }^{\mathbf{5}} \mathbf{L}^{\mathbf{6}} \mathbf{L} / \mathbf{T}$ & -9167.919533 & -9168.0403411 & -9168.0403411 \\
${ }^{\mathbf{5}} \mathbf{L}^{\mathbf{6}} \mathbf{L} / \mathbf{X}$ & -9167.9204177 & -9168.0409998 & -9168.0409997 \\
$\mathbf{L}_{\mathbf{2}} / \mathbf{H}$ & -9167.9628527 & -9168.0277433 & -9167.9996311 \\
${ }^{\mathbf{a}} \mathbf{S}^{\mathbf{b}} \mathbf{S} / \mathbf{X}$ & -9167.9180584 & -9168.0420955 & -9168.0420957 \\
${ }^{\mathbf{b}} \mathbf{S}^{\mathbf{3}} \mathbf{L} / \mathbf{H}$ & -9167.9150567 & -9168.0356 & -9168.0356 \\
\hline
\end{tabular}



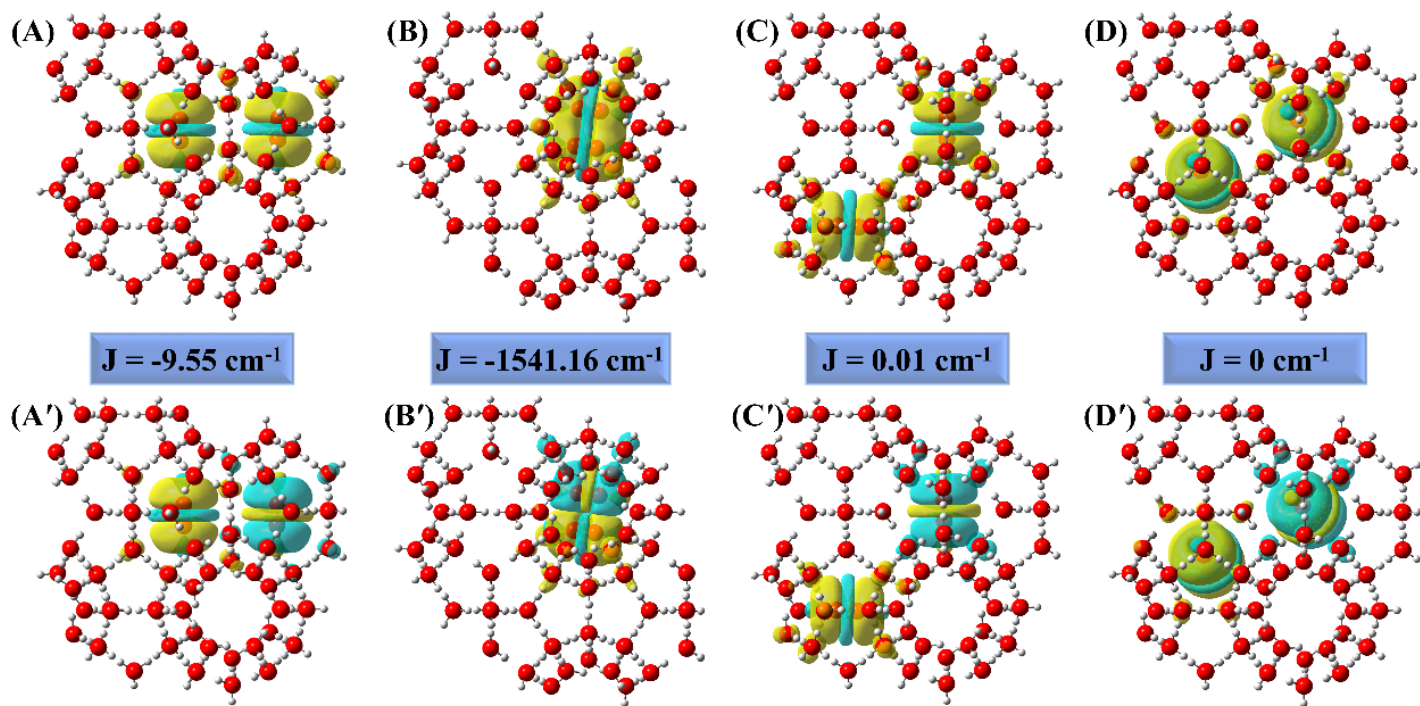

Figure S11. Spin density distributions for ${ }^{5} \mathrm{~L}^{6} \mathrm{~L} / \mathrm{H}(\mathrm{A}), \mathrm{L}_{2} / \mathrm{H}(\mathrm{B}),{ }^{\mathrm{a}} \mathrm{S}^{\mathrm{b}} \mathrm{S} / \mathrm{X}(\mathrm{C})$, and ${ }^{\text {b }} \mathrm{S}{ }^{3} \mathrm{~L} / \mathrm{H}$ (D) structures, indicating different magnetic spin coupling patterns.

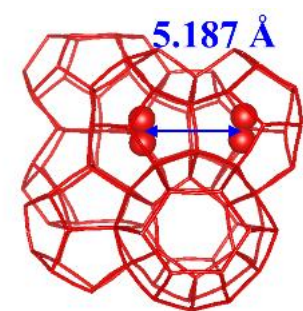

$\varepsilon=-7 \%$

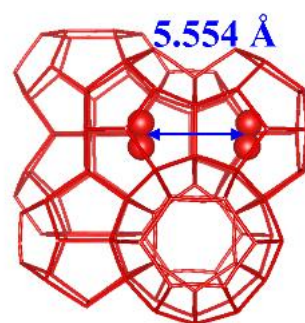

$\varepsilon=-2 \%$

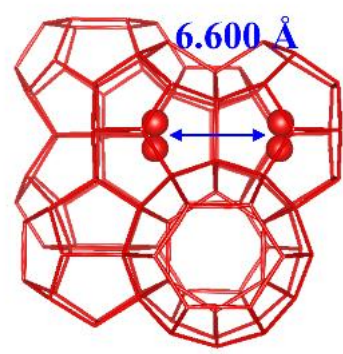

$\varepsilon=10 \%$

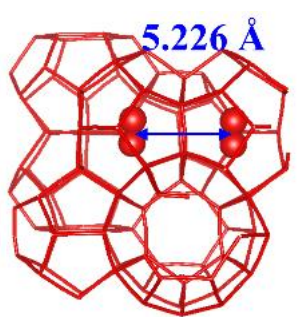

$\varepsilon=-6 \%$

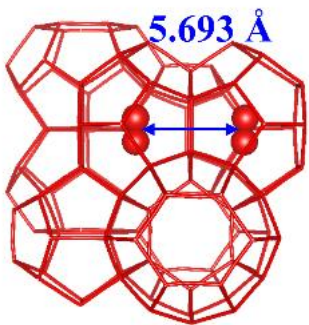

$\varepsilon=0 \%$

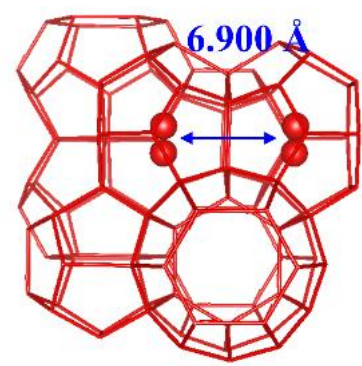

$\varepsilon=15 \%$
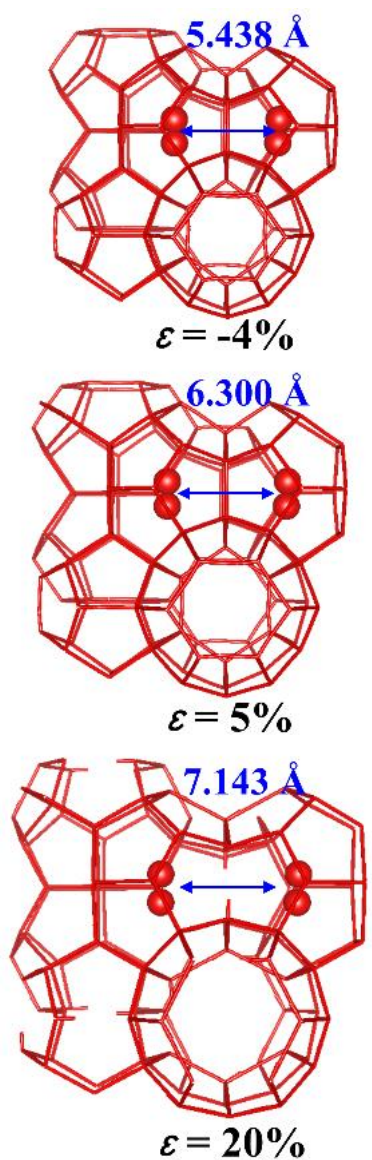

Figure S12. Stable magnetic configurations of the ${ }^{5} \mathrm{~L}^{6} \mathrm{~L}(\mathrm{H})$ structures under strain. All hydrogen atoms in the cell are omitted. 
Table S7. The Calculated $a$, Diameters of Single Large Cage $\left(D_{\mathrm{L}}\right)$, Total Energies $(\mathrm{E})$, Stable Magnetic States and Formation energies $\left(E_{\text {form }}\right)$ for the ${ }^{5} \mathrm{~L}^{6} \mathrm{~L}(\mathrm{H})$ Structures under Strain

\begin{tabular}{cccccc}
\hline $\boldsymbol{\varepsilon}(\boldsymbol{\%})$ & $\boldsymbol{a}$ & $\boldsymbol{D}_{\mathbf{L}}(\stackrel{\AA}{\mathbf{A}})$ & $\mathbf{E}(\mathbf{e V} /$ cell $)$ & $\begin{array}{c}\text { Magnetic } \\
\text { state }\end{array}$ & $\begin{array}{c}\boldsymbol{E}_{\text {form }} \\
(\mathbf{e V} / \text { cell })\end{array}$ \\
\hline-7 & 10.597 & 7.820 & -703.623 & AFM & -0.228 \\
-6 & 10.711 & 7.984 & -704.950 & AFM & -0.230 \\
-4 & 10.939 & 8.139 & -707.000 & AFM & -0.231 \\
-2 & 11.167 & 8.308 & -708.180 & AFM & -0.234 \\
0 & 11.396 & 8.472 & -708.684 & AFM & -0.236 \\
5 & 11.970 & 8.880 & -707.717 & AFM & -0.200 \\
10 & 12.540 & 9.265 & -705.095 & NM & -0.155 \\
15 & 13.110 & 9.597 & -702.013 & NM & -0.145 \\
20 & 13.680 & 9.990 & -699.034 & NM & -0.026 \\
\hline
\end{tabular}

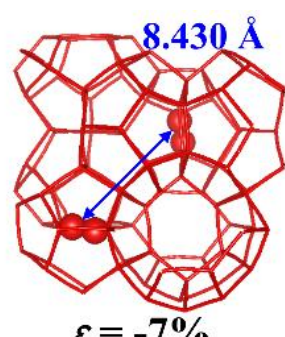

$\varepsilon=-7 \%$

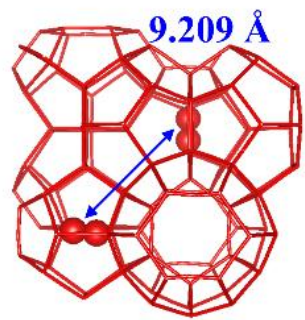

$\varepsilon=-2 \%$

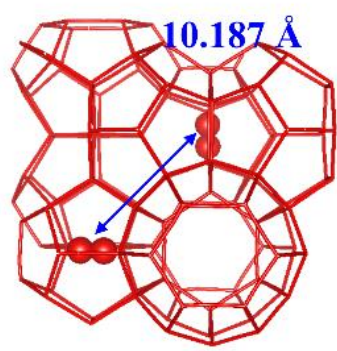

$\varepsilon=10 \%$
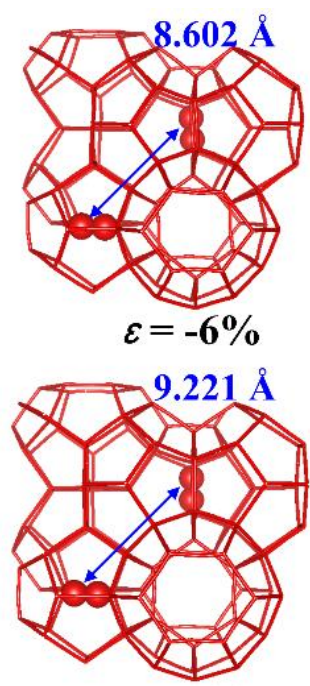

$\varepsilon=0 \%$

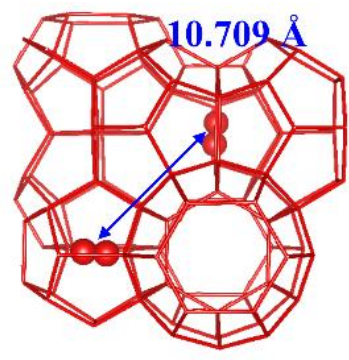

$\varepsilon=15 \%$
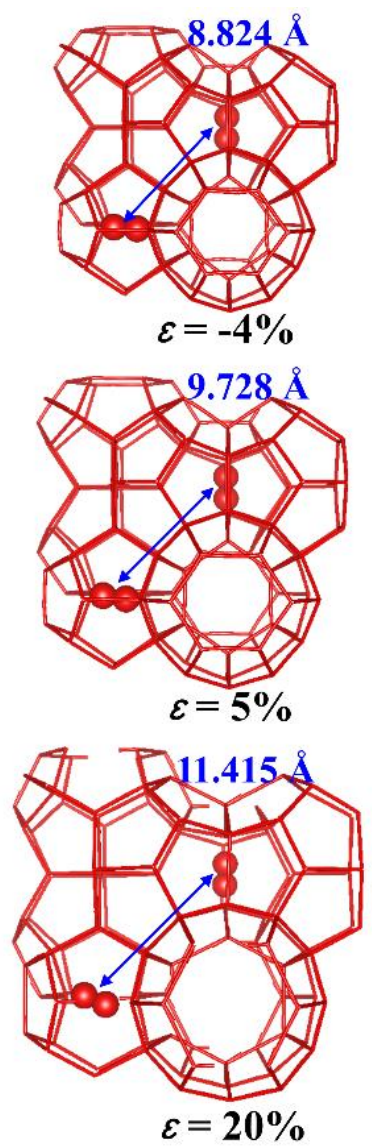

Figure S13. Stable magnetic configurations of the ${ }^{\mathrm{a}} \mathrm{S}{ }^{\mathrm{b}} \mathrm{S}(\mathrm{X})$ structures under strain. All hydrogen atoms in the cell are omitted. 
Table S8. The Calculated $a$, Diameters of Single Small Cage ( $\left.D_{\mathrm{S}}\right)$, E, Stable Magnetic States and $E_{\text {form }}$ for the ${ }^{\mathrm{a}} S^{\mathrm{b}} S(\mathrm{X})$ Structures under Strain

\begin{tabular}{cccccc}
\hline $\boldsymbol{\varepsilon}(\%)$ & $\boldsymbol{a}$ & Ds $(\AA)$ & $\begin{array}{c}\mathbf{E} \\
(\mathbf{e V} / \mathbf{c e l l})\end{array}$ & $\begin{array}{c}\text { Magnetic } \\
\text { state }\end{array}$ & $\begin{array}{c}\text { Eform } \\
(\mathbf{e V} / \mathbf{c e l l})\end{array}$ \\
\hline-7 & 10.597 & 6.878 & -703.492 & AFM & -0.097 \\
-6 & 10.711 & 6.966 & -704.845 & AFM & -0.125 \\
-4 & 10.939 & 7.128 & -706.945 & AFM & -0.176 \\
-2 & 11.167 & 7.263 & -708.173 & AFM & -0.227 \\
0 & 11.396 & 7.412 & -708.675 & PM & -0.227 \\
5 & 11.970 & 7.759 & -707.740 & FM & -0.223 \\
10 & 12.540 & 8.064 & -705.133 & FM & -0.193 \\
15 & 13.110 & 8.344 & -702.037 & FM & -0.169 \\
20 & 13.680 & 8.598 & -699.131 & FM & -0.123 \\
\hline
\end{tabular}

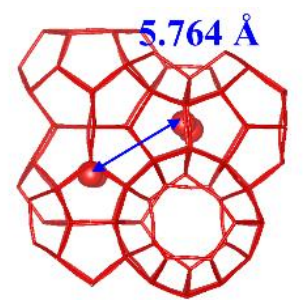

$\mathcal{E}=-\mathbf{7 \%}$

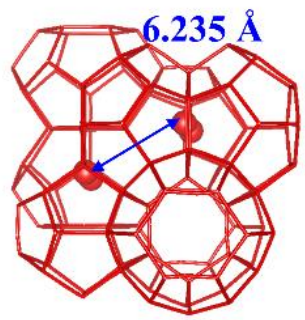

$\varepsilon=-\mathbf{2} \%$

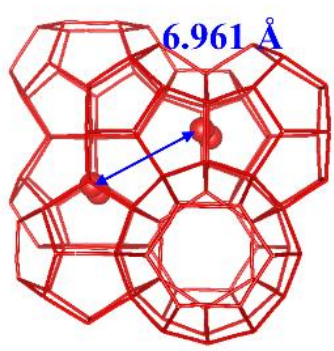

$\varepsilon=10 \%$
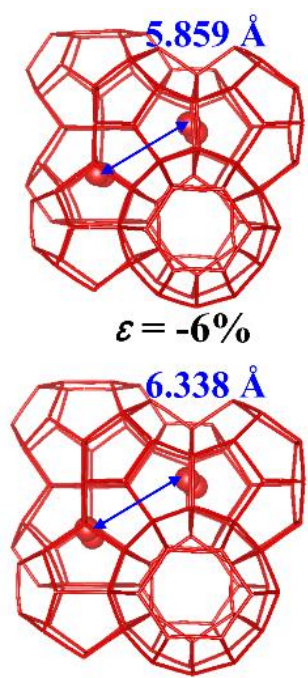

$\varepsilon=0 \%$

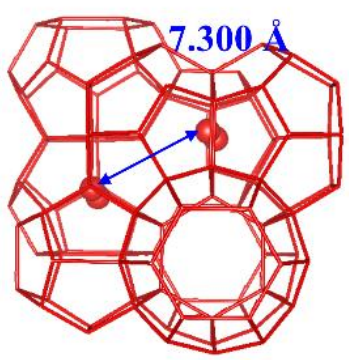

$\varepsilon=15 \%$
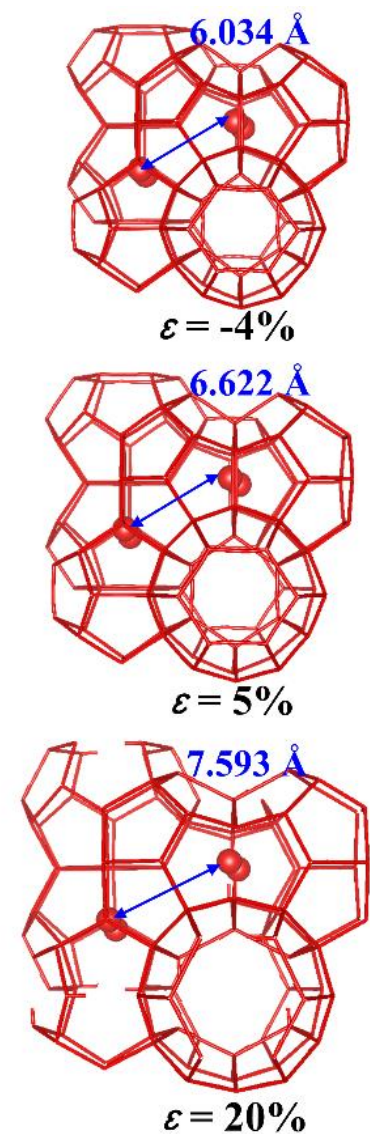

Figure S14. Stable magnetic configurations of the ${ }^{b} S^{3} L(H)$ structures under strain. All hydrogen atoms in the cell are omitted. 
Table S9. The Calculated $a, D_{\mathrm{L}}, D_{\mathrm{S}}$, E, Stable Magnetic States and $E_{\text {form }}$ for the ${ }^{\mathrm{b}} \mathrm{S}{ }^{3} \mathrm{~L}(\mathrm{H})$ Structures under Strain

\begin{tabular}{ccccccc}
\hline $\boldsymbol{\varepsilon}(\boldsymbol{\%})$ & $\boldsymbol{a}$ & $\boldsymbol{D}_{\mathbf{L}}(\mathbf{\AA})$ & Ds $(\mathbf{A})$ & $\mathbf{E}(\mathbf{e V / c e l l})$ & $\begin{array}{c}\text { Magnetic } \\
\text { state }\end{array}$ & $\begin{array}{c}\text { Eform } \\
(\mathbf{e V} / \mathbf{c e l l})\end{array}$ \\
\hline-7 & 10.595 & 7.888 & 6.923 & -703.455 & AFM & -0.060 \\
-6 & 10.709 & 7.974 & 6.999 & -704.836 & AFM & -0.116 \\
-4 & 10.937 & 8.148 & 7.143 & -706.931 & AFM & -0.162 \\
-2 & 11.165 & 8.309 & 7.267 & -708.160 & AFM & -0.214 \\
0 & 11.392 & 8.473 & 7.407 & -708.673 & PM & -0.225 \\
5 & 11.962 & 8.876 & 7.785 & -707.733 & FM & -0.216 \\
10 & 12.532 & 9.273 & 8.068 & -705.127 & FM & -0.187 \\
15 & 13.131 & 9.616 & 8.365 & -702.037 & FM & -0.169 \\
20 & 13.671 & 10.018 & 8.536 & -699.132 & FM & -0.124 \\
\hline
\end{tabular}

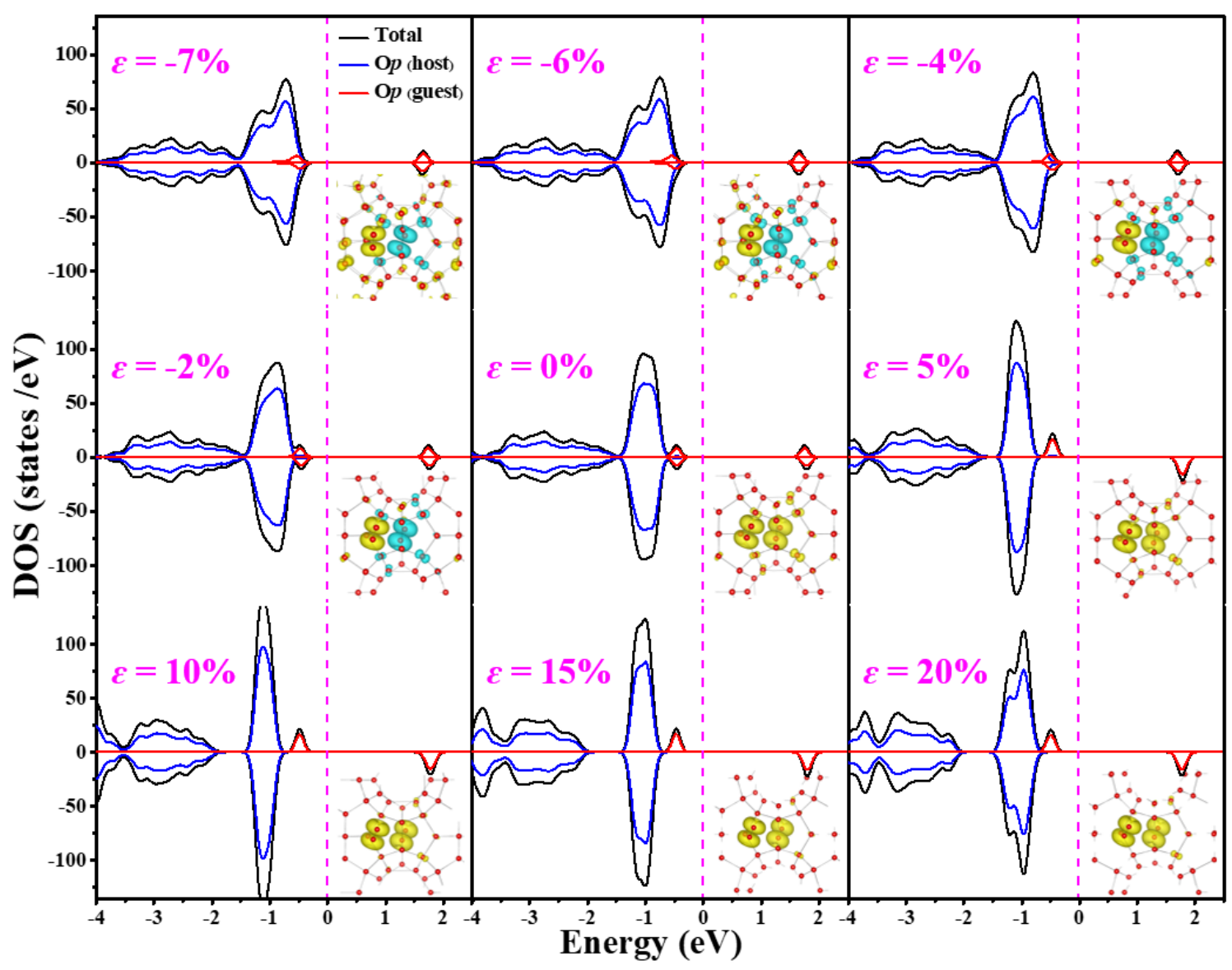

Figure S15. Total DOS and corresponding PDOS of ${ }^{\mathrm{b}} \mathrm{S}^{3} \mathrm{~L}$ structures under the strain from $-7 \%$ to $20 \%$. The vertical dashed line represents the Fermi level. The insets are corresponding spinpolarized charge density distributions. 


\section{Comparison of the DOS and PDOS between $\mathrm{O}_{2} @ \mathrm{CHs}$ and $\square @ \mathrm{CHs}$}

We add a simple discussion for the density of states (DOS) and the atomic site projected density (PDOS) between the $\mathrm{O}_{2} @ \mathrm{CHs}\left({ }^{5} \mathrm{~L}^{6} \mathrm{~L}\right.$ and $\left.{ }^{\mathrm{a}} \mathrm{S}^{\mathrm{b}} \mathrm{S}\right)$ and those after vertically removing the double $\mathrm{O}_{2}$ ( $\square @ \mathrm{CHs}$ ). As Figure S16 illustrates, the DOS distribution of $\square @ \mathrm{CHs}$ locates at a higher energy relative to $\mathrm{O}_{2} @ \mathrm{CHs}$, but both share similar distributions in reciprocal space. Especially, for the $\mathrm{O}_{2} @ \mathrm{CHs}$ systems under strain free, the host $\mathrm{O} 2 p$ states in $\square @ \mathrm{CHs}$ are shifted noticeably to the lower energy. The energy shift confirms that such clathrate hydrates are more stable due to the inclusion of $\mathrm{O}_{2}$ molecules. More particularly, the free-standing $\square @ \mathrm{CHs}$ system is nonmagnetic system without unpaired electrons, while the encapsulation of $\mathrm{O}_{2}$ molecules induces spin polarization. The valence band maximum (VBM) and conduction band minimum $(\mathrm{CBM})$ in the band structures of $\mathrm{O}_{2} @ \mathrm{CHs}$ are mainly contributed by the guest $\mathrm{O}_{2}$, as observed around the Fermi level. The magnetic configuration is found to be the most stable for the $\mathrm{O}_{2}$-encaged systems. Such magnetism mainly arises from the unpaired electrons in $\mathrm{O}_{2}$ molecules.

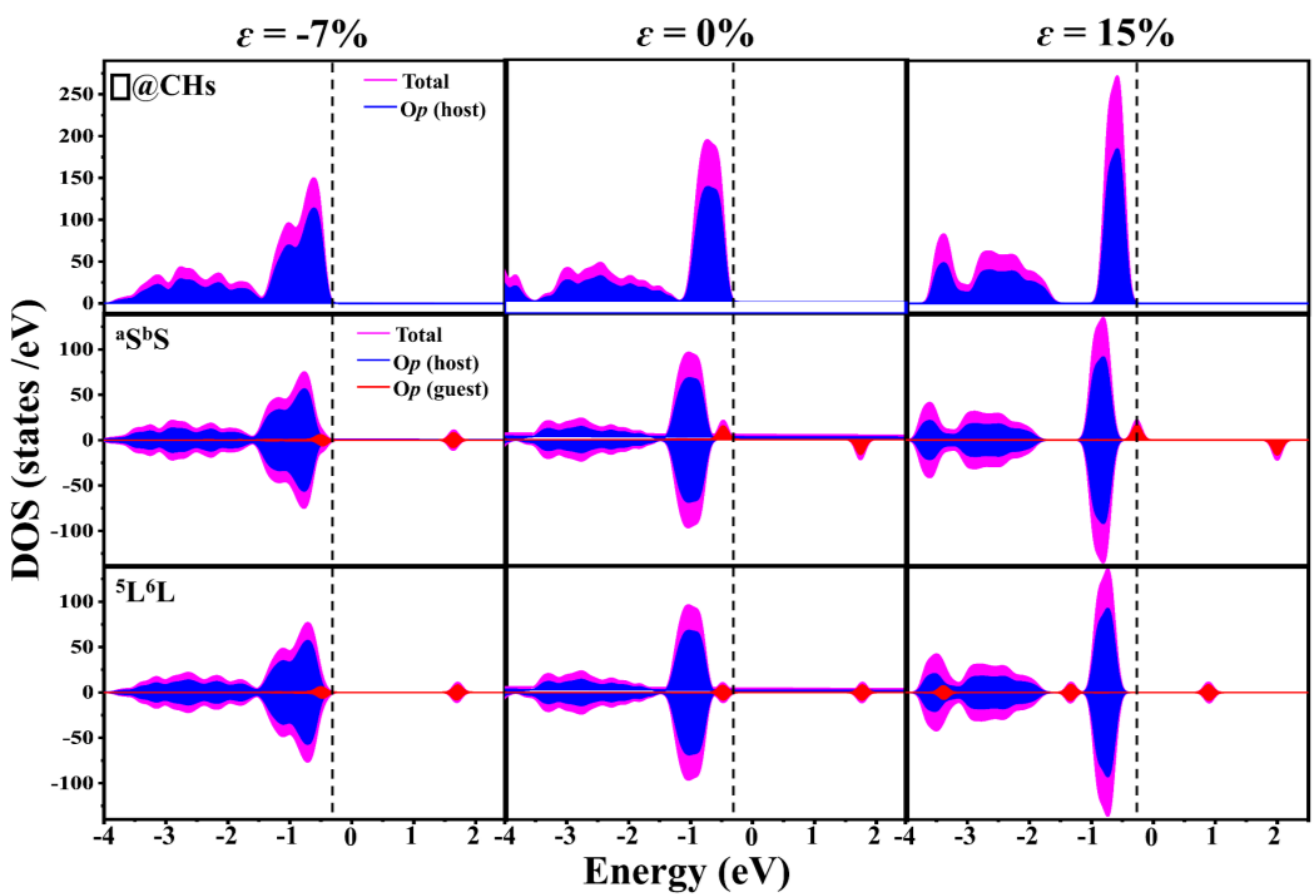

Figure S16. Total DOS and corresponding PDOS of $\mathrm{O}_{2} @ \mathrm{CHs}\left({ }^{5} \mathrm{~L}^{6} \mathrm{~L}\right.$ and $\left.{ }^{\mathrm{a}} \mathrm{S}^{\mathrm{b}} \mathrm{S}\right)$ and their $\square @ \mathrm{CHs}$ structures under $15 \%$ tension strain, strain free and $-7 \%$ compression strain. The vertical dashed line represents the valence band maximum (VBM). 


\section{Preliminary Examination of the Possibility of $\mathrm{O}_{2}$ Moving from One Cavity to Another by}

\section{through the Shared Six/Five-Membered Water Rings}

We further studied the migration of $\mathrm{O}_{2}$ between two adjacent water cages, as shown in Figure $\mathrm{S} 17$. In the $\mathrm{O}_{2}$ clathrate hydrates, the small $\left(5^{12}\right)$ and large $\left(5^{12} 6^{2}\right)$ cages share a pentagonal face and the large and large cages share a hexagonal face. The energy profiles for $\mathrm{O}_{2}$ migration between the cages are shown in Figure S17, at different distances from the outer hexagonal face of a $5^{12} 6^{2}$ cage, to its center of the $5^{12} 6^{2}$ cage, to the center of the $5^{12} 6^{2}$ or $5^{12}$ cage, and finally to near the edge of the $5^{12} 6^{2}$ or $5^{12}$ cage. The barriers of an $\mathrm{O}_{2}$ passing through the hexagonal face of a large cage and the pentagonal face of a small cage are $21.38 \mathrm{kcal} / \mathrm{mol}$ and $77.85 \mathrm{kcal} / \mathrm{mol}$, respectively, and the barriers of their reverse processes are essentially the same (21.49 and $77.71 \mathrm{kcal} / \mathrm{mol}$ through the hexagonal and pentagonal faces, respectively). The inter-cage migration studies reveal that the energy barriers exist for $\mathrm{O}_{2}$ to leave or approach the cage edges. This means that if an $\mathrm{O}_{2}$ is confined in a water cage, it is very difficult for it to escape from the cage, or approach the cage edges.

(A)

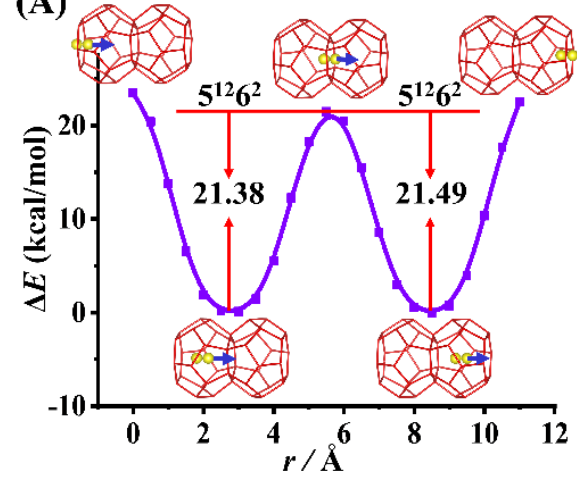

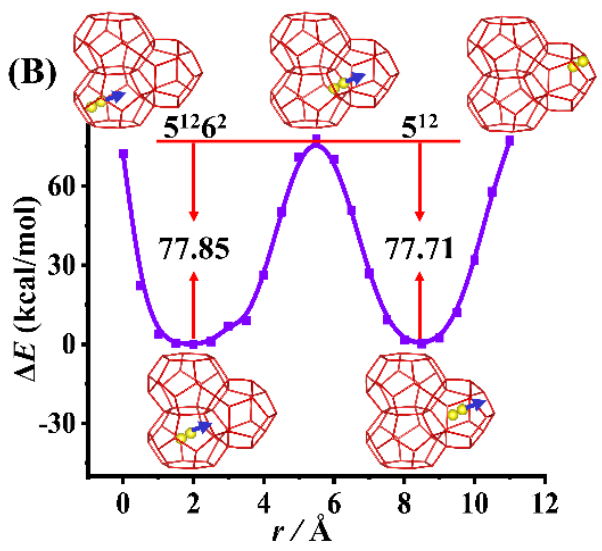

Figure S17. Energy profiles for $\mathrm{O}_{2}$ movement between cages in the $\mathrm{O}_{2}$ clathrate hydrates starting from the edge of the $5^{12} 6^{2}$ cage, to the center of pentagonal or hexagonal faces, and finally to near the edge of the $5^{12} 6^{2}$ or $5^{12}$ cage. 